\title{
The Modified Star-Ring Architecture for High-Capacity Subcarrier Multiplexed Passive Optical Networks
}

\author{
Wen-Piao Lin, Ming-Seng Kao, Member, IEEE, and Sien Chi, Fellow, OSA
}

\begin{abstract}
A modified star-ring architecture (MSRA) consisting of a star network on the upper level and many concatenated ring subnets on the lower level is proposed for subcarrier multiplexed passive optical network (SCM-PON). The upper level star network ensures high network capacity and its weakness in reliability is overcome by the concatenated ring subnets with self-healing capabilities. The self-healing function can be performed at remote nodes (RNs) by using optical switches (OS) to reconfigure the ring subnets if any link fails. Moreover, we design the cascade add/drop transceiver (CAT) structure in the lower level ring subnets to completely eliminate the optical beat interference $(\mathrm{OBI})$ problem and achieve an OBI-free optical network. Finally, we set up an experimental network to demonstrate the feasibility of this cost-effective survivable structure.
\end{abstract}

Index Terms-Cascade add/drop transceiver (CAT), modified star-ring architecture (MSRA), optical beat interference (OBI), reliability, self-healing function.

\section{INTRODUCTION}

$\mathbf{S}$ UBCARRIER multiplexed passive optical networks (SCM-PONs) are cost-effective and favorable architectures for subscriber networks such as CATV and FTTH [1]-[3]. In densely populated commercial and residential areas, the number of nodes may increase significantly and a high-capacity network is necessary under this circumstances. The network has to be reliable as well, which can be recovered from failure condition within a short period of time [4].

The optical beat interference (OBI) could seriously impair the SCM-PON, especially when the number of nodes is large. It will degrade the carrier-to-noise ratio (CNR) and limit the number of optical network units (ONUs) [5]-[7]. Many valuable approaches were proposed to reduce the influence of OBI. For instance, Woodward et al. suggested that different mode spacing lasers could effectively reduce OBI [8]. Feldman et al. demonstrated that the penalty of OBI could be eliminated by amplified LEDs in a subcarrier multiple access system [9]. Besides, our previous work showed that a strong sine wave modulation could reduce OBI to an acceptable level [10]. However, all of the methods mentioned above have treated the OBI problem individually at each optical node. As the number of nodes increases, not only does the OBI problem become severe but the excess ca-

Manuscript received May 8, 2000; revised September 13, 2000

W.-P. Lin and S. Chi are with the Institute of Electro-Optical Engineering, National Chiao-Tung University, Hsinchu 30056, Taiwan, R.O.C.

M.-S. Kao is with the Department of Communication Engineering, National

Chiao-Tung University, Hsinchu 30056, Taiwan, R.O.C.

Publisher Item Identifier S 0733-8724(01)00409-1. pacity plus the tolerance to faults also decrease. This will make the network not capable of providing an acceptable quality of service. Therefore, we shall suggest a novel approach to solve these problems effectively.

In this paper, we propose a modified star-ring architecture (MSRA) which employs a properly optimized two-level network architecture having a star network on the upper level along with many concatenated ring subnets on the lower level. A special CAT structure is employed at each mode to eliminate the OBI problem. This architecture is a feasible choice in terms of the capacity, the OBI-reducing capability, the quality-of-service (QoS), and the cost of initial installation. We had analyzed the performance of the MSRA and carried out an experimental network to demonstrate its feasibility.

The rest of this paper is organized as follows. Section II introduces the MSRA in detail and Section III analyzes its performance. In Section IV, we implement an experimental network and demonstrate its experimental results. Finally, Sections V and VI are devoted to the discussion and conclusions of this study.

\section{THE MOdIFIED STAR-RING ARCHITECTURE}

The most common physical topologies of customer access networks from a central office (CO) to subscribers are star, ring, and bus topologies. The star topology is attractive due to the fact that the transfer from a narrowband network to a broad-band customer access network is easy and effective. If the number of subscribers is increased, the star network can be easily broken up into several subnets, being a versatile and flexible architecture for network expansion.

Among all of the subcarrier multiplexed passive optical networks, the two-level star-star architecture is popular because it owns low propagation delay, low first installed cost and high compatibility. But if the network is large, its reliability and the inherent OBI problems become significant. To overcome the weakness of star networks while preserving their advantages, we propose a novel network architecture, as shown in Fig. 1. The proposed MSRA is based on the two-level star-ring architecture with special arrangements on the lower level ring subnets.

In the MSRA, downstream signals originating from the $\mathrm{CO}$ are distributed to the remote nodes (RNs) via the upper level star network, and then are further delivered to ONUs through the lower level ring subnets. On the other hand, upstream signals generated at each $\mathrm{ONU}$ are transmitted to the $\mathrm{RN}$ responsible 


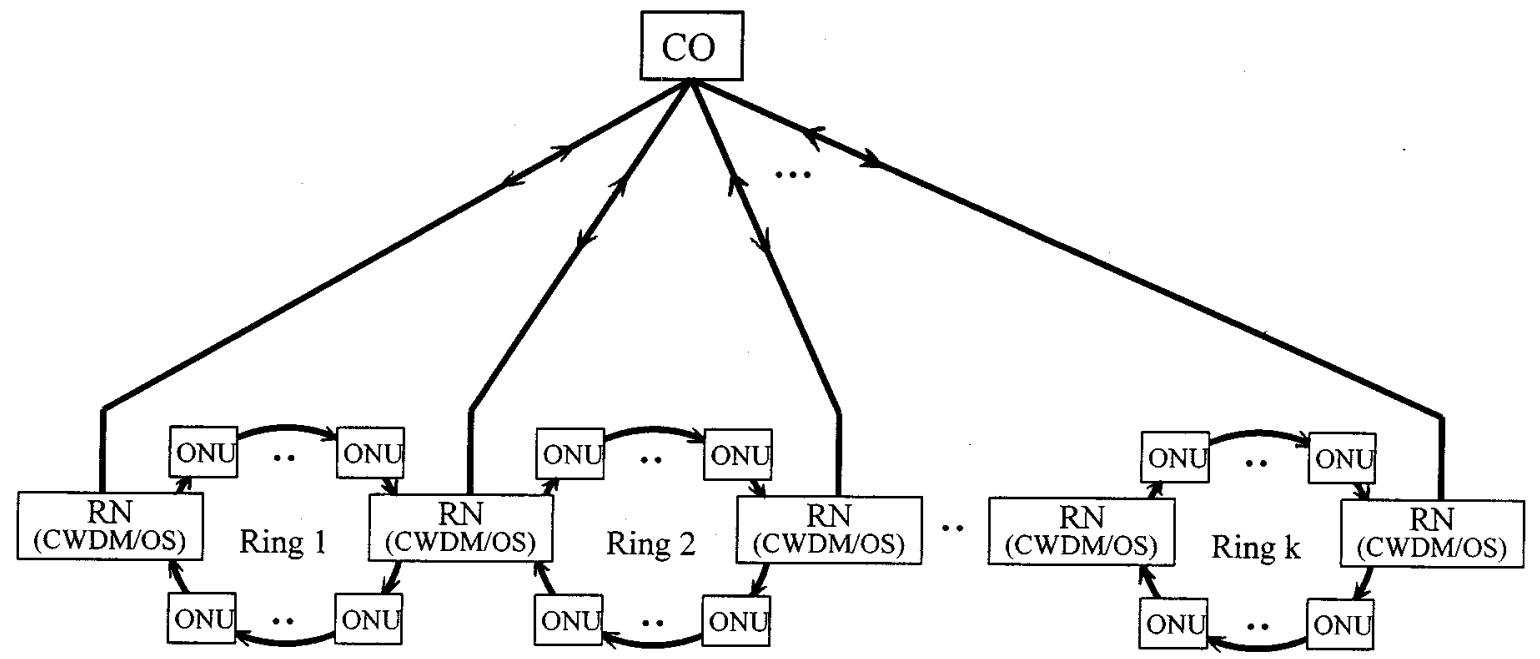

Fig. 1. Block diagram of the modified star-ring architecture (MSRA).

for the ONU of interest via the lower level ring subnet, and then further delivered to the $\mathrm{CO}$ using the upper level star network. The $1.55-\mu \mathrm{m}$ band is used for downstream transmission and the 1.3- $\mu \mathrm{m}$ band for upstream transmission.

Every lower level ring subnet is divided into two semirings by RNs, with each consisting of several ONUs. The function of $\mathrm{RN}$ is to properly transfer signals between the $\mathrm{CO}$ and the lower level ring subnet in the normal condition, and to perform self-healing function if link failure occurs in the upper level star network. As shown in Fig. 2, each RN contains two $1.55-\mu \mathrm{m} / 1.3 \mu \mathrm{m}$ coarse wavelength-division multiplexers (CWDMs) and several optical switches (OS). A signaling tone is constantly sent by the $\mathrm{CO}$ and detected by the control circuit in the RN. The switch states are normally set as shown in Fig. 2(a), therefore the $1.55-\mu \mathrm{m}$ downstream signals and the $1.3-\mu \mathrm{m}$ upstream signals are properly forwarded by the two CWDMs. If the corresponding link in the upper level star network is broken, the signaling tone will be absent and the control circuit will reconfigure the OS functioning as Fig. 2(b) such that the upstream signals can bypass the $\mathrm{RN}$ of interest and go to the neighboring RNs. In this scenario, the network is virtually reconfigured, as shown in Fig. 3, such that the affected ONUs will be assisted by the two neighboring RNs. Obviously, the downstream signals can still be transmitted from the $\mathrm{CO}$ to the affected ONUs.

In each ONU, we design the CAT structure to overcome the OBI problem. Please refer to Fig. 4 to understand the idea of CAT. In the $\mathrm{ONU}_{1}$, the downstream optical signal originated from the $\mathrm{CO}$ is first converted to electrical by a p-i-n module. Then this signal is forwarded to an add/drop module in which the upstream signal $\left(S_{1}\right)$ is added and the message destined to the $\mathrm{ONU}_{1}\left(C_{1}\right)$ is dropped. The output of the add/drop module, which contains the downstream signal from the $\mathrm{CO}$ and the upstream signal of the $\mathrm{ONU}_{1}$, is transmitted to the next ONU by a 1.3- $\mu \mathrm{m}$ laser transmitter. The same operation is performed in the following ONUs in a cascade manner. Thus, all the downstream signals can be received by the destined ONUs and all the upstream signals can be transferred to the RN, and then to the

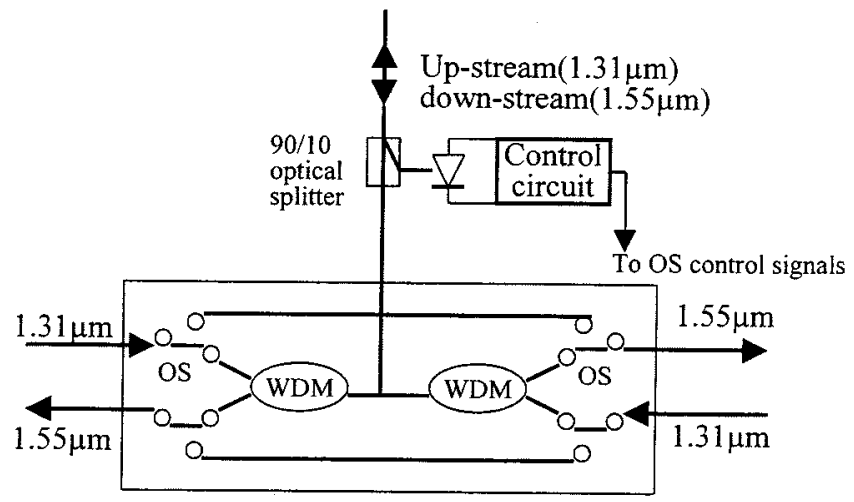

(a)

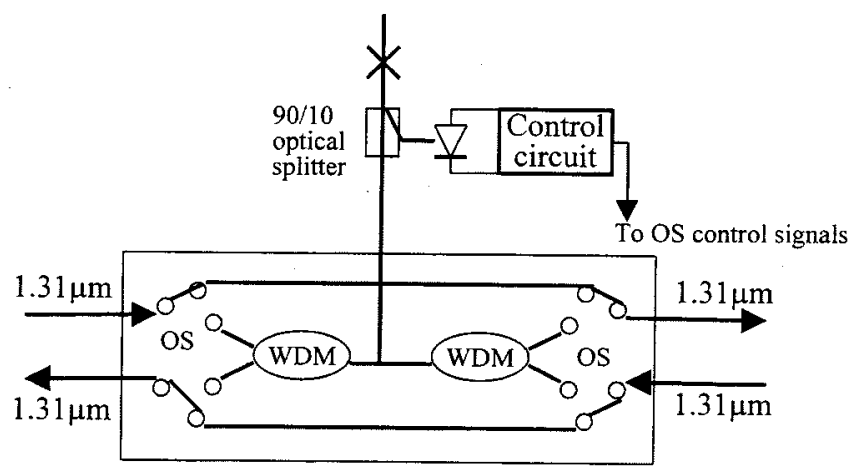

(b)

Fig. 2. Connections of remote node for (a) normal operations and (b) fiber branch or optical component failures.

$\mathrm{CO}$ afterwards. The CAT structure indeed brings two major advantages.

- Since the upstream optical signals come from a sole laser transmitter all the time, there is no OBI problem in the 


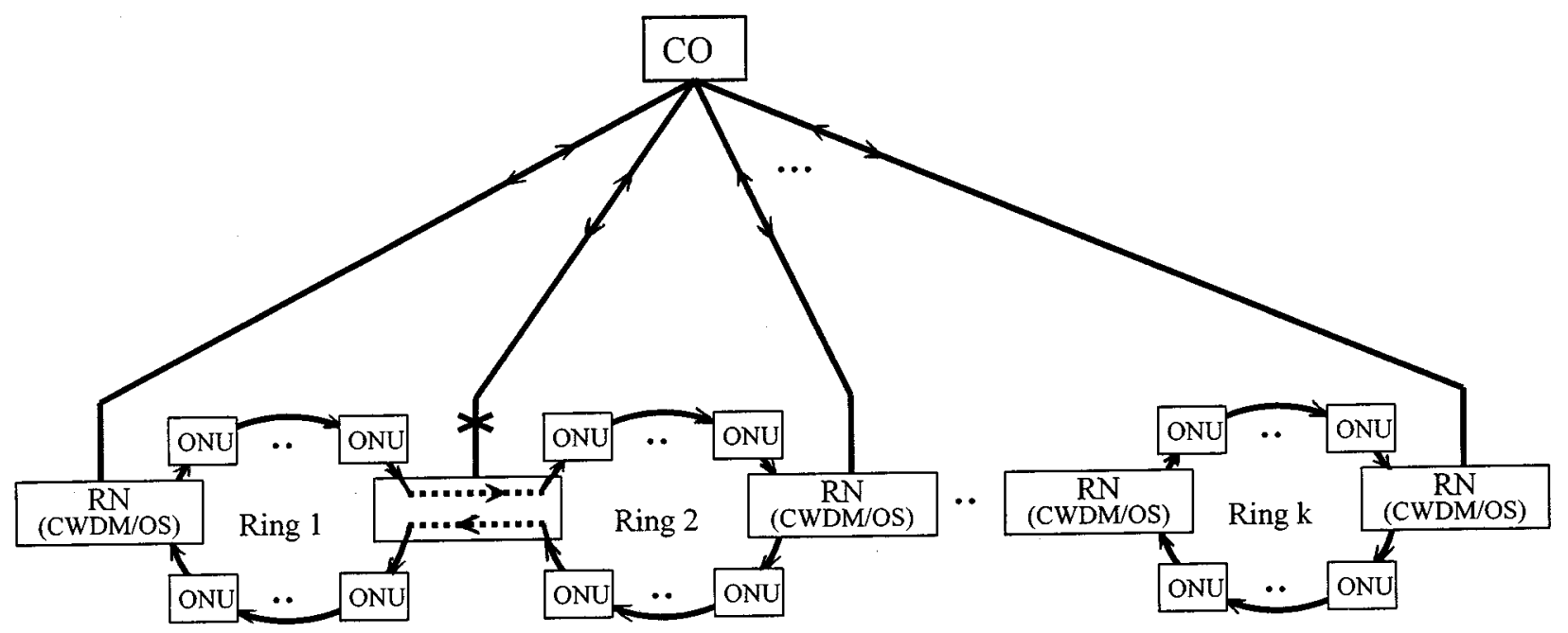

Fig. 3. Virtual configuration of the lower level ring under link failure on the upper level star network.

lower level ring subnets, and in turn the whole network is OBI free.

- The downstream/upstream signals are regenerated at each ONU, so that low-power lasers can be used and there is no branch loss at each ONU. This is not the case for other systems in which optical amplifiers are usually needed to compensate for branch losses for upstream/downstream signals.

The detail configuration of ONU in our experiments to be demonstrated later is shown in Fig. 5. The electrical signals from the p-i-n module output include the downstream signal in the high-frequency band and the upstream signals in the low-frequency band. Using a band-pass filter (BPF), a demultiplex (DMUX), and a demodulator (DFSK), the downstream signal (digital data or multi-channel video signals) can be dropped. On the other hand, a local upstream signal is added to the $\mathrm{p}$-i-n module output before modulating a $1.3-\mu \mathrm{m}$ laser. The laser output is sent to the next ONU. In each ONU we use a pair of OS to bypass the node when it fails.

In general, the modulated frequency bandwidth of a commercial Fabry-Perot (FP) laser diode is about $1.0 \mathrm{GHz}$. In the MSRA, the downstream signal frequency bandwidth ranges from $550 \mathrm{MHz}$ to $1.0 \mathrm{GHz}$ and the upstream signal frequency bandwidth is from $80 \mathrm{MHz}$ to $500 \mathrm{MHz}$, respectively. As each branch of the upper level star network can use the full modulation bandwidth to serve the corresponding ONUs, the MSRA can provide very high-capacity information transfer and serve a large number of subscribers.

\section{PeRformance AnAlysis}

The signal quality of subcarrier networks is closely related to the CNR. The CNR is related to the optical modulation index as well as the system noise. In the CAT structure, since the distance between ONUs is short as normally, the receiver noise can be ignored due to large optical power output. Thus in general laser source noise dominates.

\section{A. CNR of the Upstream Signal}

Referring to Fig. 4, the output signal power $P_{0}$ in the p-i-n module at the $\mathrm{CO}$ for a number of $k$ cascade ONUs is given as

$$
\begin{aligned}
P_{0}=\{ & {\left[\left(S_{1}+n_{1}\right) \cdot G_{1} \cdot \ell_{1} \cdot R_{2} \cdot A_{2}+n_{2}+S_{2}\right) } \\
& \left.\cdot G_{2} \cdot \ell_{2} \cdot R_{3} \cdot A_{3}+S_{3}+n_{3}\right] \\
& \left.\cdot G_{3} \cdot \ell_{3} \cdot R_{4} \cdot A_{4}+\cdots+S_{k}+n_{k}\right\} \\
& G_{k} \cdot \ell_{k} \cdot R_{k+1} \cdot A_{k+1} \cdot+n_{k+1}
\end{aligned}
$$

where

$S_{i} \quad$ upstream signal power;

$n_{i} \quad$ total noise power;

$G_{i} \quad$ E/O conversion gain of the laser transmitter;

$\ell_{i} \quad$ transmission loss factor;

$R_{i} \quad$ responsivity of the $\mathrm{p}$-i-n diode;

$A_{i} \quad$ gain of the receiver preamplifier for the $i$ th ONU, respectively.

Note that here the $\mathrm{CO}$ is taken as the $(k+1)$ th ONU for brevity. The loss factor $\ell_{i}$ is given as

$$
\ell_{i}=e^{-\alpha L_{i}}
$$

where $\alpha$ is the fiber loss coefficient; and $L_{i}$ the fiber length between $\mathrm{ONU}_{i}$ and $\mathrm{ONU}_{i+1}$. The upstream signals $S_{i}$ are subcarrier multiplexed at different frequency bands and they are independent of each other. Without loss of generality, we assume $G_{i} \cdot \ell_{i} \cdot R_{i+1} \cdot A_{i+1} \cong 1, i=1 \sim k$ then the $\mathrm{CNR}_{i}$ for each subcarrier signal at the receiver output of $\mathrm{CO}$ is given as

$$
\begin{aligned}
\mathrm{CNR}_{i} & =\frac{C_{i}}{P_{\text {noise }}}=\frac{S_{i}}{\sum_{i=1}^{k+1} n_{i}} \\
& =\frac{\frac{1}{2} m_{i}^{2} I_{0}^{2}}{k \cdot\left(\mathrm{RIN} \cdot I_{0}^{2} \cdot B+2 q I_{0} B+4 K T B F / R_{e q}\right)}
\end{aligned}
$$




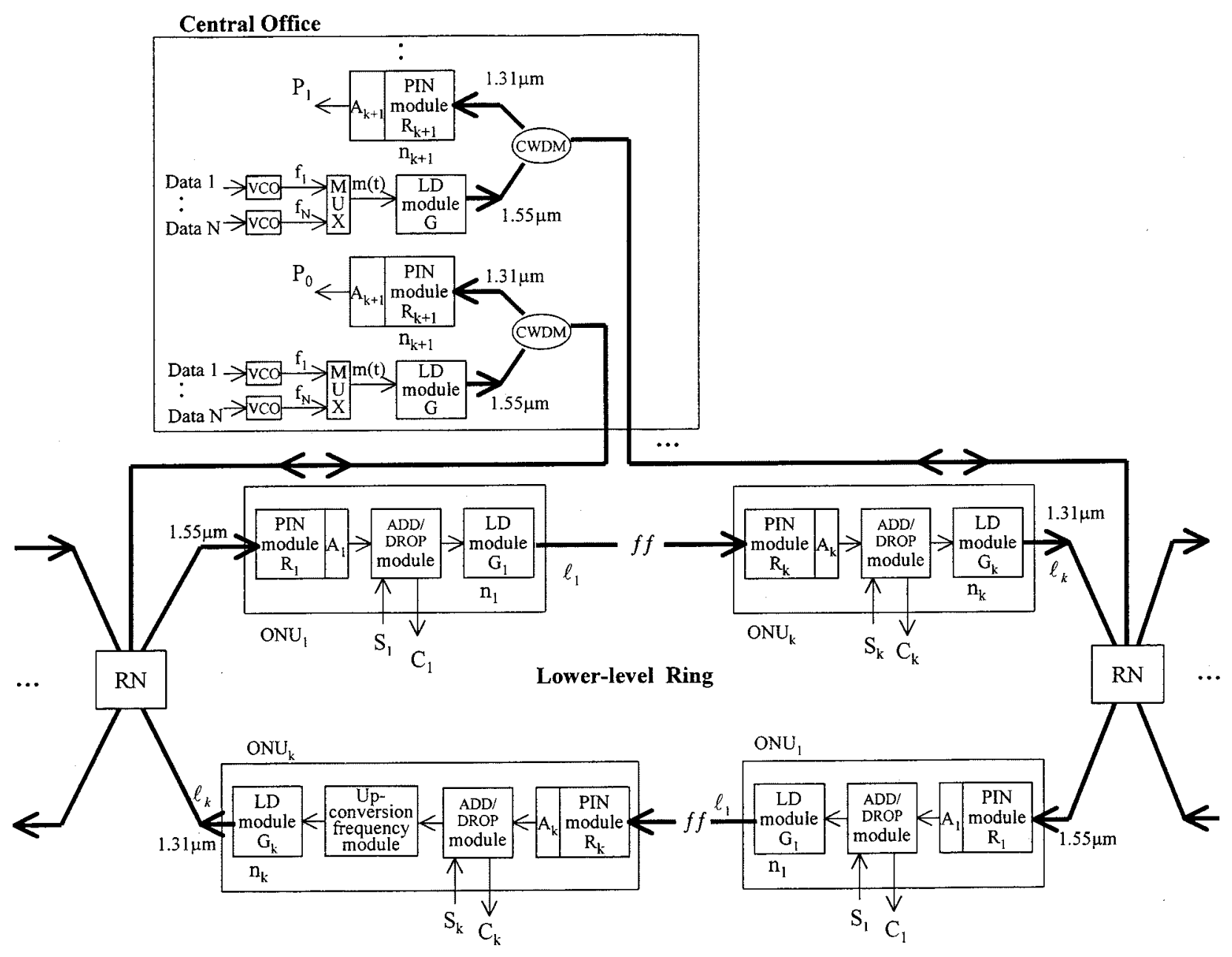

Fig. 4. The cascade add/drop transceiver (CAT) structure in the lower level ring.

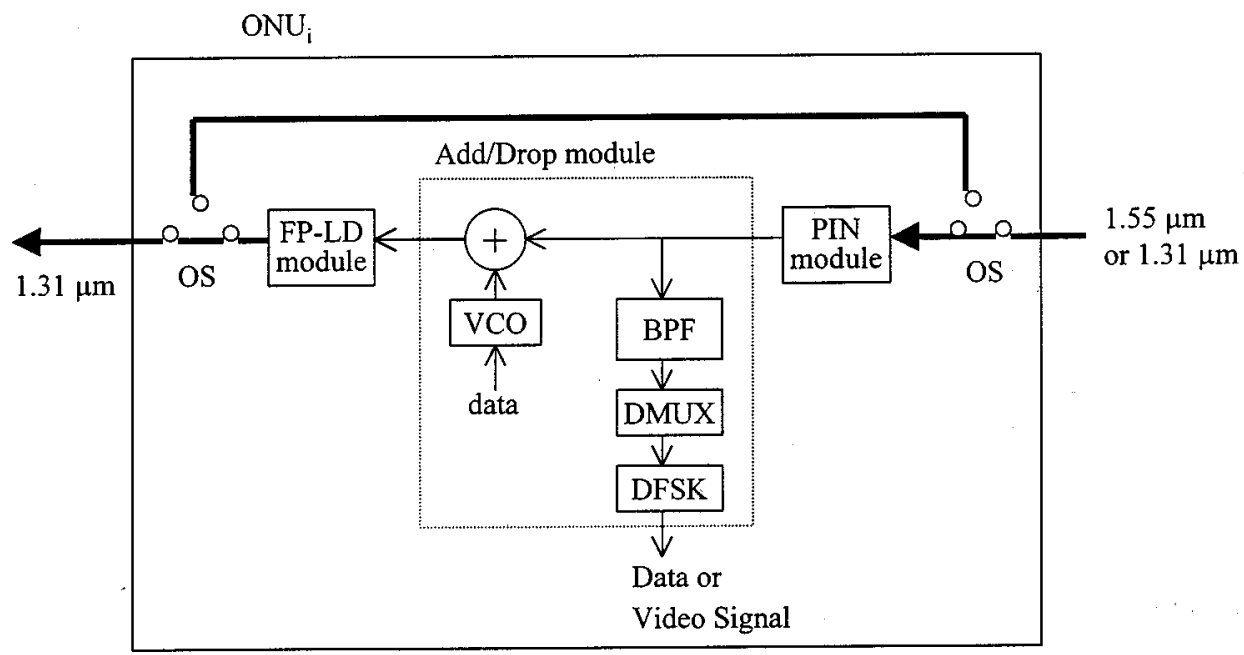

Fig. 5. Detailed configuration of ONU.

where $I_{0}$ is the average photocurrent and $m_{i}$ the optical modulation index per channel corresponding to $S_{i}$. RIN is the relative intensity noise of the laser source, $B$ the bandwidth of each channel. The shot noise $2 q I_{0} B$ and the thermal noise $4 K T B F / R_{e q}$ are produced by the receiver, with $R_{e q}$ being the equivalent resistance and $F$ the noise figure. Note that $n_{1}$ consists of LD noise and $n_{k+1}$ has receiver noise only.

As shown in Fig. 1, the upstream signals in each branch of the upper level star network come from cascade ONUs from two semirings in the lower level. If the two semirings have the 
same number as $k$ ONUs, the $\mathrm{CNR}_{i}$ is written as (4), shown at the bottom of the page.

\section{B. CNR of the Upstream Signal Under Link Failure}

If a link in the upper level star network is broken, those ONUs originally served by the RN connected to the broken link will be served by the two neighboring RNs as shown in Fig. 3. In this case, the number of ONUs served by the neighboring $\mathrm{RN}$ is increased. If a cluster of $u$ links are failed simultaneously and there are $k$ ONUs in each lower level semiring, it is uncomplicated to obtain the CNR as in (5), shown at the bottom of the page.

\section{CNR of the Downstream Signal}

As shown in Fig. 4, the downstream signal from the $\mathrm{CO}$ is received and regenerated at each ONU. As additional noise is added at each ONU, the CNR of the downstream signal of the $\mathrm{ONU}_{1}$ will be the highest and that of the $\mathrm{ONU}_{k}$ will be the worst. Using previous results, it is easy to obtain the CNR at the $j$ th $\mathrm{ONU}$ as

$$
\begin{aligned}
\mathrm{CNR}_{j} & =\frac{S_{j}}{n_{c o}+\sum_{i=1}^{j} n_{i}} \\
& =\frac{\frac{1}{2} m_{j}^{2} I_{0}^{2}}{j \cdot\left(\mathrm{RIN} \cdot I_{0}^{2} \cdot B+2 q I_{0} B+4 K T B F / R_{e q}\right)}
\end{aligned}
$$

where $n_{c o}$ is the $\mathrm{LD}$ noise at the $\mathrm{CO}$, and $n_{j}$ consists of receiver noise only.

\section{Results}

Fig. 6 shows the value of CNR in the upstream direction versus the maximum number of cascade ONUs in each ring. If there is no failure, the number of maximum cascade ONUs in each ring can reach up to 78 in the present case when CNR $=$ $17 \mathrm{~dB}, m=0.032, I_{0}=0.9 \mathrm{~mA}, T=300 \mathrm{~K} B=6 \mathrm{MHz}$, $F=3$ and RIN $=-135 \mathrm{~dB} / \mathrm{Hz}$. When the failed linking branch $u=1$, the maximum number of cascade ONUs will be reduced to 52 . Moreover, as $u=2$, the maximum number of cascade ONUs is only 39 . If RIN is reduced to $-145 \mathrm{~dB} / \mathrm{Hz}$, the maximum number of cascade ONUs is 118 under a normal working condition. In the event that the number of failed branches $u=1$ and $u=2$, the number of cascade ONUs can change to 78 and 56 , respectively. The results reveal that RIN is a critical parameter in the current system.

RIN is indeed not the only critical parameter in the system if the number of active ONUs is large. In this case nonlinear distortion induced by laser clipping should be taken into consideration. In general, merely part of the ONUs are active such that the total number of upstream and downstream channels will be lower than 150 (there are about 75 upstream channels and 75 downstream channels in the proposed frequency band). Even under the worst case $N=150$, by carefully choosing the OMI/ch, a high C/NLD still could be achieved [11].

\section{EXPERIMENTAL SETUP AND RESULTS}

To demonstrate the feasibility of the MSRA, we setup an experimental network as shown in Fig. 7. In the downstream direction, a commercial $1.55-\mu \mathrm{m}$ FP laser is directly modulated by a composite signal consisting of several $3 \mathrm{Mb} / \mathrm{s}$ FSK subcarrier signals and analog FM video channels. The FSK channels are $6 \mathrm{MHz}$ apart, and the spacing for FM video channels is 38.36 MHz. The multiplexer frequency bandwidth covers 550-1000 $\mathrm{MHz}$. In the upstream direction, there are eight ONUs forming two rings in the network to be linked to $\mathrm{CO}$ via three branches of a $8 \times 8$ star coupler. A low-cost $1.3-\mu \mathrm{m}$ FP laser in the $\mathrm{ONU}_{i}$ is directly modulated by several $3 \mathrm{Mb} / \mathrm{s}$ FSK subcarrier signals originated from the $(i-1)$ cascade ONUs ahead of the $\mathrm{ONU}_{i}$. The upstream FSK subcarrier channel spacing is $6 \mathrm{MHz}$, ranging from 80 to $500 \mathrm{MHz}$. The $3 \mathrm{Mb} / \mathrm{s}$ data signal can be converted into an FSK signal through a VCO module with a modulation index of 0.5. And the output of FSK signals is used to directly modulate the laser having an optical modulation index of 0.2 . In this work, the upstream subcarrier channels were chosen from $94 \mathrm{MHz}$ to $136 \mathrm{MHz}$, and the $106-\mathrm{MHz}$ channel $\left(\mathrm{ONU}_{3}\right)$ is monitored during this experiment. In the downstream direction, te subcarrier channels were chosen from $550 \mathrm{MHz}$ to $580 \mathrm{MHz}$, and the $556 \mathrm{MHz}$ subcarrier channel is monitored. The total optical loss through the $2.2 \mathrm{~km}$ single-mode fiber is $0.82 \mathrm{~dB}$ at $1.3 \mu \mathrm{m}$ and $0.43 \mathrm{~dB}$ at $1.55 \mu \mathrm{m}$, respectively. The end-to-end

$$
\mathrm{CNR}_{i}=\frac{S_{i}}{P_{\text {noise }}}=\frac{S_{i}}{n_{k+1}+2 \sum_{i=1}^{k} n_{i}}=\frac{\frac{1}{2} m_{i}^{2} I_{0}^{2}}{2 k \cdot\left(\mathrm{RIN} \cdot I_{0}^{2} \cdot B\right)+(2 k-1) \cdot\left(2 q I_{0} B+4 K T B F / R_{e q}\right)} .
$$

$$
\mathrm{CNR}_{i}=\frac{S_{i}}{P_{\text {noise }}}=\frac{S_{i}}{n_{k+1}+(u+2) \sum_{i=1}^{k} n_{i}}=\frac{\frac{1}{2} m_{i}^{2} I_{0}^{2}}{(u+2) \cdot k \cdot\left(\mathrm{RIN} \cdot I_{0}^{2} \cdot B\right)+[(u+2) \cdot k-1] \cdot\left(2 q I_{0} B+4 K T B F / R_{e q}\right)}
$$




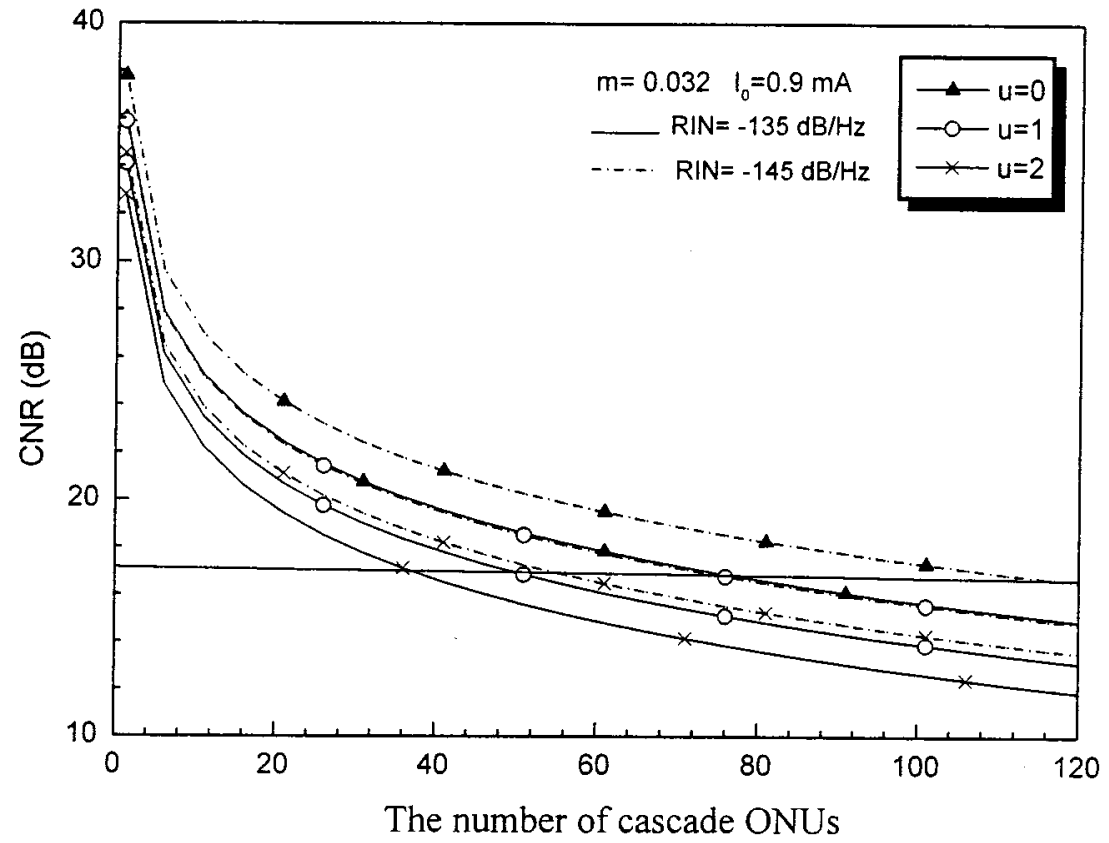

Fig. 6. CNR versus the number of cascade ONUs in each ring. For the normal operation $u=0$ and the branch failures $u=1$ and 2 under $m=0.032, I_{0}=0.9$ $\mathrm{mA}, B=6 \mathrm{MHz}, T=300^{\circ} \mathrm{K}, F=3, R_{e q}=50 \Omega$ and $\mathrm{RIN}=-135 \mathrm{~dB} / \mathrm{Hz}$ and $-145 \mathrm{~dB} / \mathrm{Hz}$.

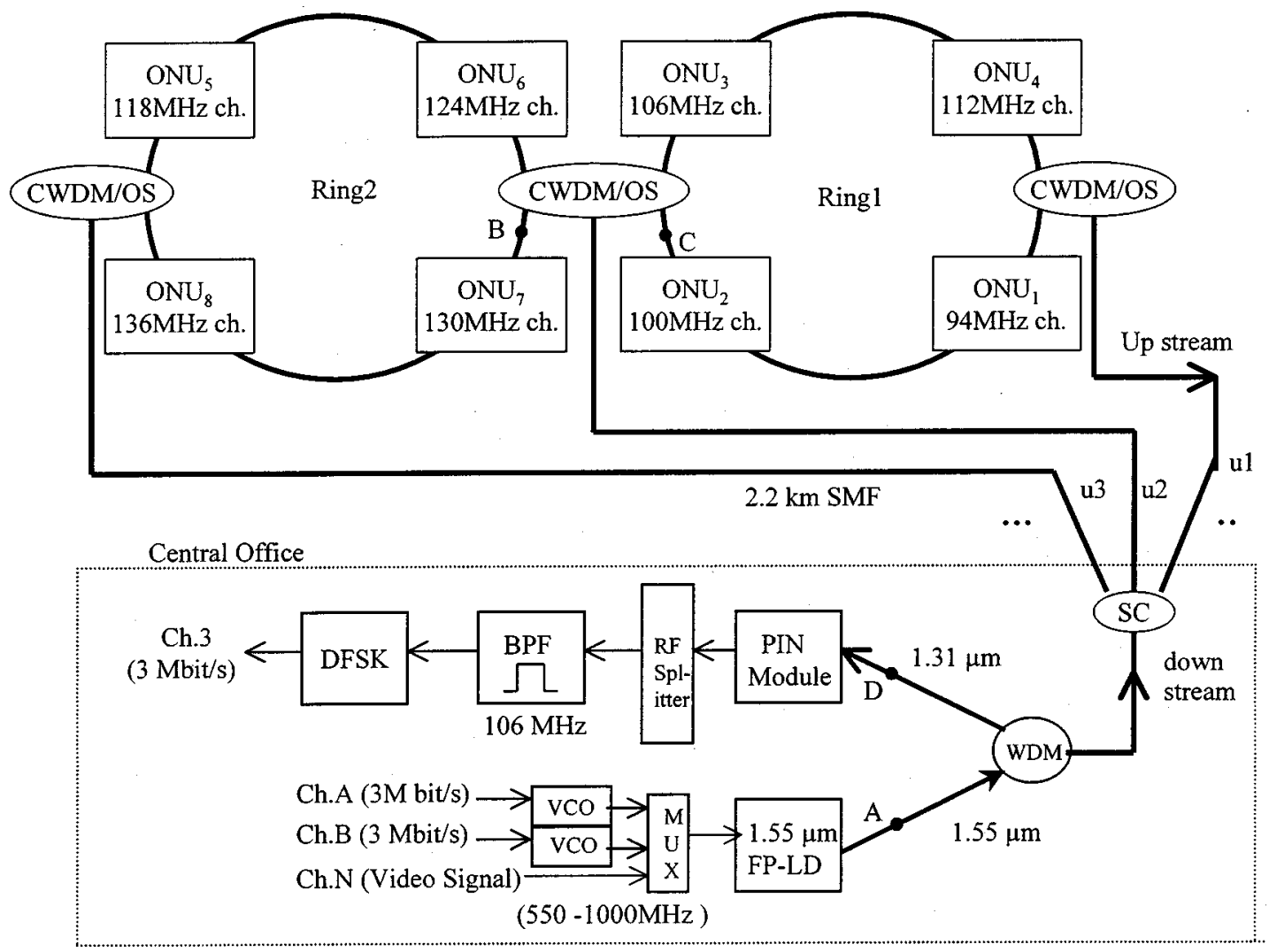

Fig. 7. Experimental setup.

optical loss of the system is $13.76 \mathrm{~dB}$ at $1.55 \mu \mathrm{m}$ (from Point A to $\mathrm{B}$ ) and $14.34 \mathrm{~dB}$ at $1.3 \mu \mathrm{m}$ (from Point $\mathrm{C}$ to $\mathrm{D}$ ).

Fig. 8 shows the measured BER of the $556 \mathrm{MHz}$ downstream channel versus the received optical power at the output of the p-i-n module in the $\mathrm{ONU}_{3}$. It is monitored under four different cases:

1) transmit the $556 \mathrm{MHz}$ channel only;

2) add two adjacent downstream SCM channels; 
3) add two adjacent downstream SCM channels and eight SCM channels in the upstream direction;

4) the same as case 3 except as branch $u 2$ fails.

For the 1.55- $\mu \mathrm{m}$ FP laser at the CO, the transmitted optical power is $-4 \mathrm{dBm}$ and a BER of $10^{-9}$ is obtained at a received optical power of $-26.4 \mathrm{dBm}$ in case 1 . There are 0.4 and $1.6 \mathrm{~dB}$ power penalties due to the eight upstream channels in cases 3 and 4 , respectively. Thus, the downstream channel optical power margin is $8.24 \mathrm{~dB}(-4-13.76-0.4+26.4)$ in case 2 , and 7.04 $\mathrm{dB}(-4-13.76-1.6+26.4)$ when branch u2 fails.

In Fig. 9, the $106 \mathrm{MHz}$ upstream channel is monitored under the following four situations:

1) transmit the $106 \mathrm{MHz}$ channel only;

2) add seven adjacent upstream SCM channels;

3) add three downstream channels and seven adjacent upstream SCM channels;

4) same as case 3 except as branch $u 2$ fails.

For the 1.3- $\mu \mathrm{m}$ FP laser in $\mathrm{ONU}_{2}$, the transmitted optical power is $-4.5 \mathrm{dBm}$, and a BER of $10^{-9}$ is obtained at a received optical power of $-29.3 \mathrm{dBm}$ in case 1 . There is a $0.7 \mathrm{~dB}$ power penalty with seven adjacent SCM channels in case 2. Moreover, a downstream channel and seven adjacent channel transmission result in $0.8 \mathrm{~dB}$ power penalty, and $1.8 \mathrm{~dB}$ power penalty when branch $\mathrm{u} 2$ fails. Thus, the $\mathrm{ONU}_{6}$ optical power margin is $9.66 \mathrm{~dB}(-4.5-14.34-0.8+29.3)$ in case 3 and $8.66 \mathrm{~dB}$ $(-4.5-14.34-1.8+29.3)$ in case 4 when branch u2 fails.

\section{DISCUSSION}

Figs. 8 and 9 reveal that the optical power penalty is less than $2 \mathrm{~dB}$ for the bidirectional transmission with eight upstream channels in the two lower level ring subnets and one downstream channel. Cascading many ONUs in the ring subnets and having several channels transmit on the downstream direction simultaneously can cause inter-channel interference (ICI) and noise accumulation problems at the p-i-n module output. Thus, a bottleneck would occur and both the transmission rate and the maximum number of cascade ONUs would be limited.

The maximum number of ONUs in the ring subnet is limited by

1) accumulated noise in each ONU;

2) the modulation bandwidth of the laser.

The limitation imposed by accumulated noises had been analyzed before. The laser modulation bandwidth is obviously a dominant factor for the maximum number of ONUs. In our system, the upstream signals are added at each ONU so that the maximum number of ONUs will be limited by the laser bandwidth in the last ONU before the RN.

In SCM-PONs, the OBI noise in the star architecture is proportion to $j(j-1) / 2$ term in the case of $j$ simultaneous optical carriers [5]. According to our previous experimental results, the system can be extended to 23 ONUs at most with a strong modulation signal when $\mathrm{CNR}=17 \mathrm{~dB}$ is required for digital FSK transmission [10]. In the CAT structure with the same lasers and $\mathrm{p}$-i-n diodes, the noise accumulation is proportion to $j\left(\mathrm{RIN}^{*} I_{0}^{2} B+2 q I_{0} B+4 K T B F / R_{e q}\right)$ term. From (6), the capacity of each ring can be up to 78 ONUs when CNR =

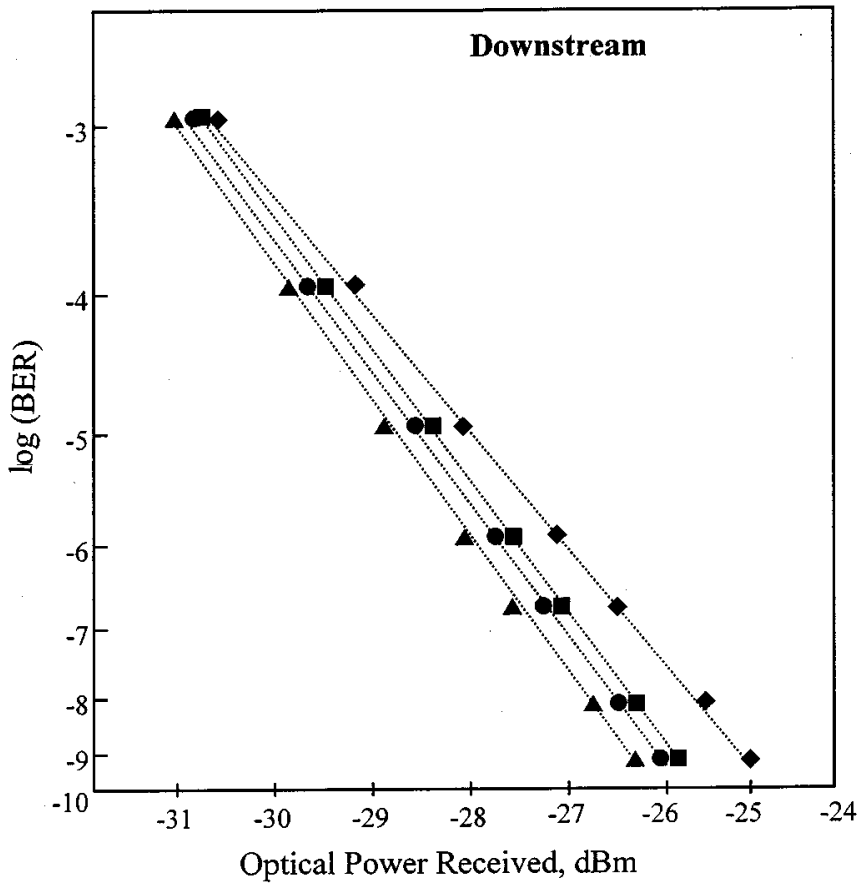

Fig. 8. Measured BER curves for downstream transmission at the $\mathrm{ONU}_{3} \boldsymbol{\Delta}$ with the $556 \mathrm{MHz}$ SCM channel only, • with two adjacent downstream SCM channels, $\mathbf{w}$ with eight upstream SCM channels and two adjacent downstream SCM channels, with eight upstream SCM channels and two adjacent downstream SCM channels if branch u2 fails.

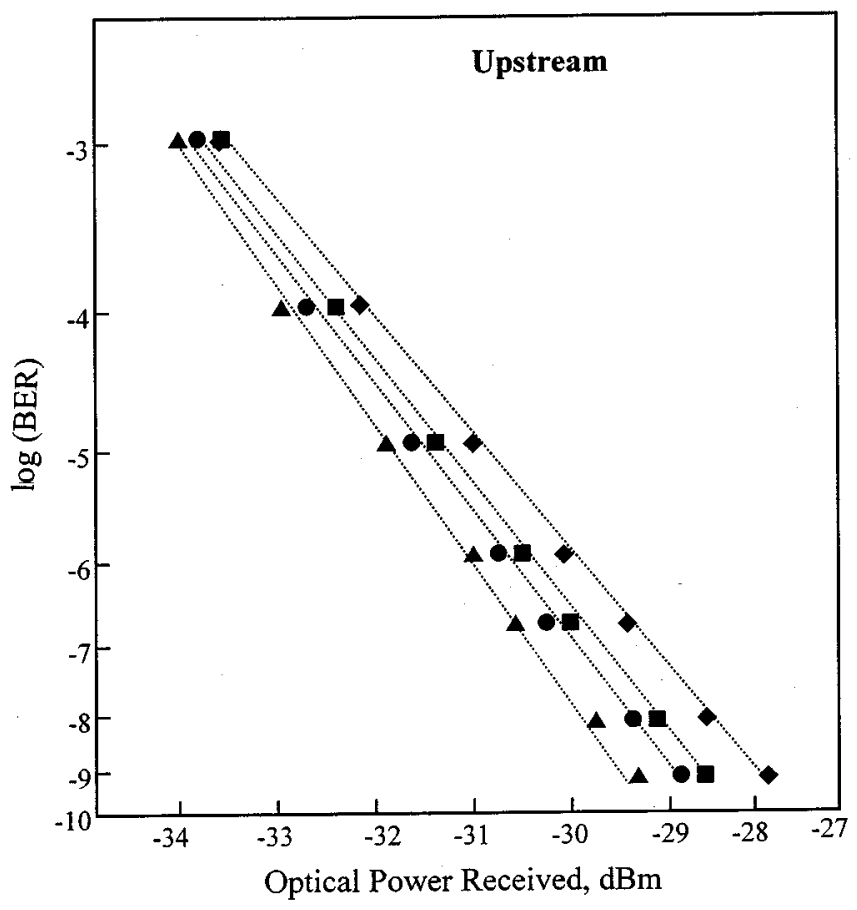

Fig. 9. Measured BER curves for upstream transmission at $\mathrm{CO} \boldsymbol{\Delta}$ with the $106 \mathrm{MHz}$ SCM channel only, $\bullet$ with seven adjacent upstream SCM channels, with three downstream SCM channels and seven adjacent upstream SCM channels. with three downstream SCM channels and seven adjacent upstream SCM channels if branch u2 fails.

$17 \mathrm{~dB}, m=0.032, I_{0}=0.9 \mathrm{~mA}$ and $\mathrm{RIN}=-135 \mathrm{~dB} / \mathrm{Hz}$, being superior to the former approach. 
In the upstream direction, as the number of subcarrier signals is increased sequentially in the succeeding ONUs, it is therefore prudent to decrease the modulation index accordingly in order to avoid nonlinear distortions. As for the downstream direction, since multichannel signals simultaneously modulate a laser, the nonlinear problem can be solved if high-linearity FP laser diode with an optical isolator and a predistortion circuit is chosen.

\section{CONCLUSION}

We have proposed and demonstrated a novel modified star-ring architecture with the cascade add/drop transceiver structure in the lower level ring subnets to obtain an OBI-free high-capacity SCM-PONs. The upper level star network ensures high network throughput and the inherent weakness of star network is adequately resolved by the concatenated lower level ring subnets with self-healing capabilities. Because of the CAT structure, the optical signals are repeatedly regenerated at each ONU and always come from a sole laser source, thereby the OBI problem can be completely eliminated. In summary, the MSRA distinct from other designs is a good candidate for future high-capacity optical networks in terms of its reliability and flexibility.

\section{REFERENCES}

[1] I. Yamashita, "The latest FTTH technologies for full service access networks," in IEEE Asia Pacific Conf. Circuits and Systems, Nov. 1996, pp. $18-21$.

[2] A. Memiya, T. Maekawa, K. Haga, and N. Tamaki, "Low cost FTTH system based on PDS architecture," in Proc. IEEE GLOBECOM, vol. 1, 1997, pp. 878-882.

[3] T. H. Wood, R. D. Feldman, and J. A. Stiles, "FiberVista: A cost-effective fiber-to-the-home (FTTH) system providing broad-band data over cable modems along with analog and digital video," IEEE Photon. Technol. Lett., vol. 114, pp. 475-477, Apr. 1999.

[4] T.-H. Wu and R. C. Lau, "A class of self-healing ring architectures for SONET network application," IEEE Trans. Commun., vol. 40, no. 11, pp. 1746-1756, Nov. 1992.

[5] C. Desem, "Optical interference in subcarrier multiplexed system with multiple optical carriers," IEEE J. Select. Areas Commun., vol. 8, pp. 1290-1295, Sept. 1990.

[6] T. H. Wood and N. K. Shankaranarayanan, "Operation of a passive optical network with subcarrier multiplexing in the presence of optical beat interference," J. Lightwave Technol., vol. 11, pp. 1632-1640, Oct. 1993.

[7] R. D. Feldman, T. H. Wood, G. Raybon, and R. F. Austin, "Effect of optical beat interference on the dynamic range of a subcarrier multiple access passive optical network using Fabry-Perot lasers," J. Lightwave Technol., vol. 14, pp. 711-715, May 1996.

[8] S. L. Woodward, X. Lu, T. E. Darcie, and G. E. Bodeep, "Reduction of optical-beat interference in subcarrier networks," IEEE Photon. Technol. Lett., vol. 8, no. 5, pp. 649-696, May 1996.
[9] R. D. Feldman, K.-Y. Liou, G. Raybon, and R. F. Austin, "Reduction of optical beat interference in a subcarrier multiple-access passive optical network through the use of an amplified light-emitting diode," IEEE Photon. Technol. Lett., vol. 8, no. 1, pp. 116-118, Jan. 1996.

[10] W.-P. Lin, "Reducing multiple optical carries interference in broadband passive optical networks," IEEE Photon. Technol. Lett., vol. 9, pp. 368-370, Mar. 1997.

[11] N. J. Frigo, M. R. Phillips, and G. E. Bodeep, "Clipping distortion in lightwave CATV systems: Models, simulations, and measurement," $J$. Lightwave Technol., vol. 11, pp. 138-146, Jan. 1993.

Wen-Piao Lin was born in Changhua, Taiwan, R.O.C., in 1957. He received the B.S. and M.S. degrees in electrical engineering from the National Taiwan Institute of Technology, R.O.C., in 1982 and 1985, respectively. He is currently pursuing the Ph.D. degree at the National Chiao-Tung University, R.O.C., with research interests in high-speed WDM networks.

From 1985 to 1987, he was with the Hua-Eng Company, Kaohsiung, Taiwan. He has been engaged in the research on optical fiber subscriber loop transmission system. In 1987, he joined the Faculty of Chien-Kuo Institute of Technology, Changhua, Taiwan, where he is now an Associate Professor.

Ming-Seng Kao (S'89-M'90) was born in Taipei, Taiwan, R.O.C., in 1959. He received the B.S.E.E. degree from National Taiwan University in 1982, the M.S. degree in optoelectronics from the National Chiao-Tung University in 1986, and the $\mathrm{Ph} . \mathrm{D}$. degree in electrical engineering from National Taiwan University in 1990.

From 1986 to 1987, he was an Assistant Researcher at the Telecommunications Laboratories, Chung-Li, Taiwan. In 1990, he joined the Faculty of National Chiao-Tung University, where he is now a Professor of Communication Engineering Department. Between 1993 and 1994, he was a Visiting Professor at the Swiss Federal Institute of Technology (ETH), Zurich, Switzerland, where he worked in the area of optical communications. He is currently interested in high-speed optical networks.

Sien Chi received the B.S.E.E. degree from the National Taiwan University and the M.S.E.E. degree from the National Chiao-Tung University, Taiwan, R.O.C., in 1959 and 1961, respectively, and the Ph.D. degree in electrophysics from the Polytechnic Institute of Brooklyn, NY, in 1971.

He joined the Faculty of National Chiao-Tung University, where he is currently a Professor of electrooptical engineering and the Vice President of the university. From 1972 to 1973, he chaired the Department of Electrophysics; from 1973 to 1977, he directed the Institute of Electronics; from 1977 to 1978, he was a Resident Visitor at Bell Laboratories, Holmdel, NJ; from 1985 to 1988, he was the Principal Advisor with the Hua-Eng Company; and from 1988 to 1990, directed the Institute of Electro-Optical Engineering. His research interests are optical fiber communications, optical solutions, and optical fiber amplifiers.

Dr. Chi received the Distinguished Research Award sponsored by the National Science Council, Taiwan in 1993, 1994, 1995, and 1996. He was the symposium chair of the International Symposium of Optoelectronics in Computers, Communications, and Control in 1992, which was co-organized by the National Chiao-Tung University and SPIE. Since 1996, he has been the Chair Professor of the Foundation for Advancement of Outstanding Scholarship. He is a Fellow of the Optical Society of America (OSA) and the PSCA. 